\title{
GAN-based Deep Neural Networks for Graph Representation Learning
}

\author{
Ming Zhao ${ }^{1}$ and yinglong zhang ${ }^{1}$ \\ ${ }^{1}$ Minnan Normal University
}

February 16, 2022

\begin{abstract}
Graph representation learning has attracted increasing attention in a variety of applications that involve learning on nonEuclidean data. Recently, generative adversarial networks(GAN) have been increasingly applied to the field of graph representation learning, and large progress has been made. However, most GAN-based graph representation learning methods use adversarial learning strategies directly on the update of the vector representation instead of the embedding mechanism, which does not make full use of the essential advantages of GAN. The essential advantage of GAN is the final embedding mechanism rather than the embedding representation itself. To address this problem, we propose to use adversarial idea on the reconstruction mechanism of deep autoencoders. Specifically, the generator and the discriminator are the two basic components of the GAN structure. We use the deep autoencoder as the discriminator, which can capture the highly non-linear structure of the graph. In addition, the generator another generative model is introduced into the adversarial learning system as a competitor. A series of empirical results proved the effectiveness of the new approach.
\end{abstract}

\section{Hosted file}

wileyNJD-AMA.bib available at https://authorea.com/users/460752/articles/556623-gan-baseddeep-neural-networks-for-graph-representation-learning

\section{Hosted file}

DnnGAN-ENGR.tex available at https://authorea.com/users/460752/articles/556623-gan-baseddeep-neural-networks-for-graph-representation-learning

\section{Hosted file}

DnnGAN-ENGR.pdf available at https://authorea.com/users/460752/articles/556623-gan-baseddeep-neural-networks-for-graph-representation-learning 

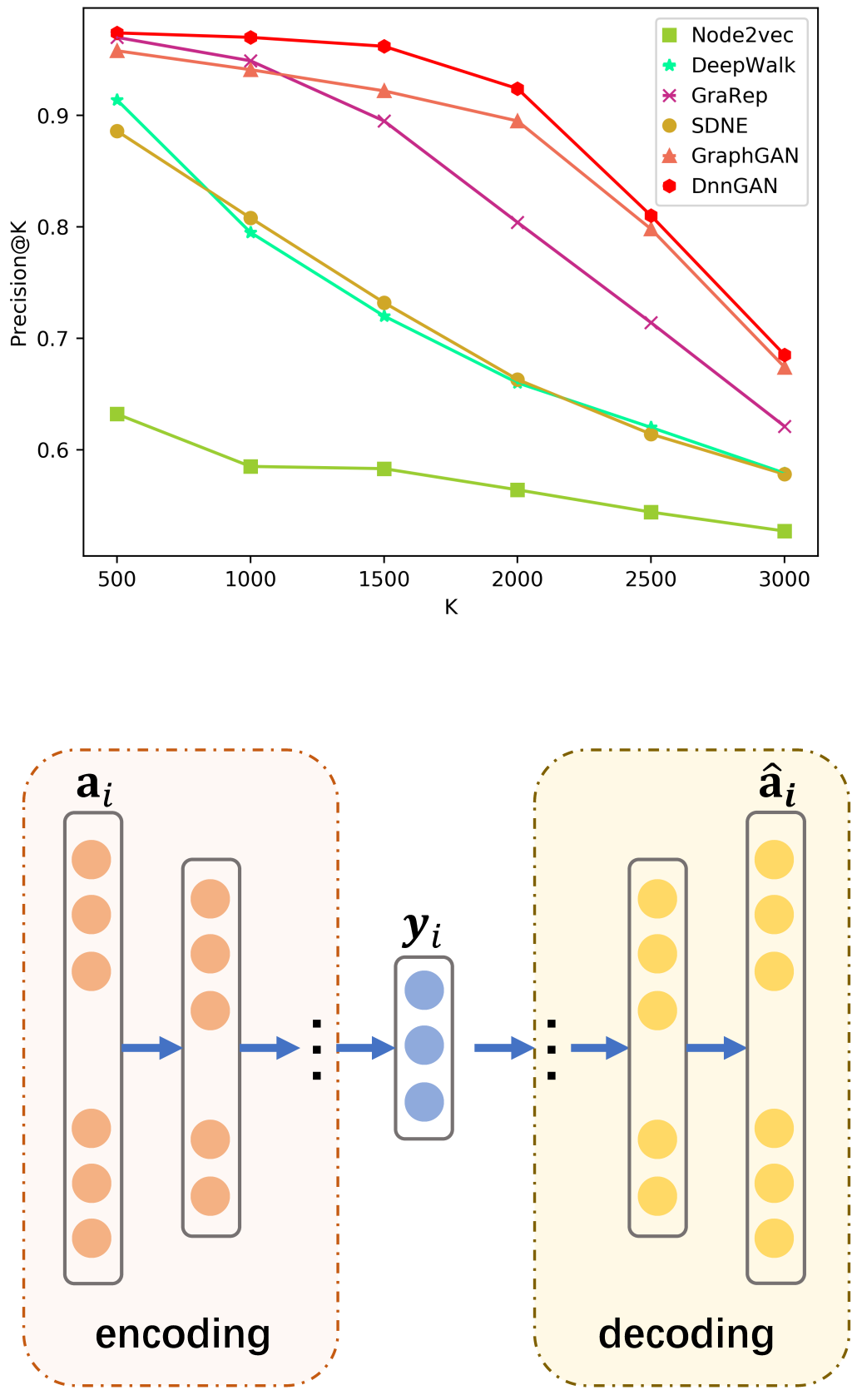

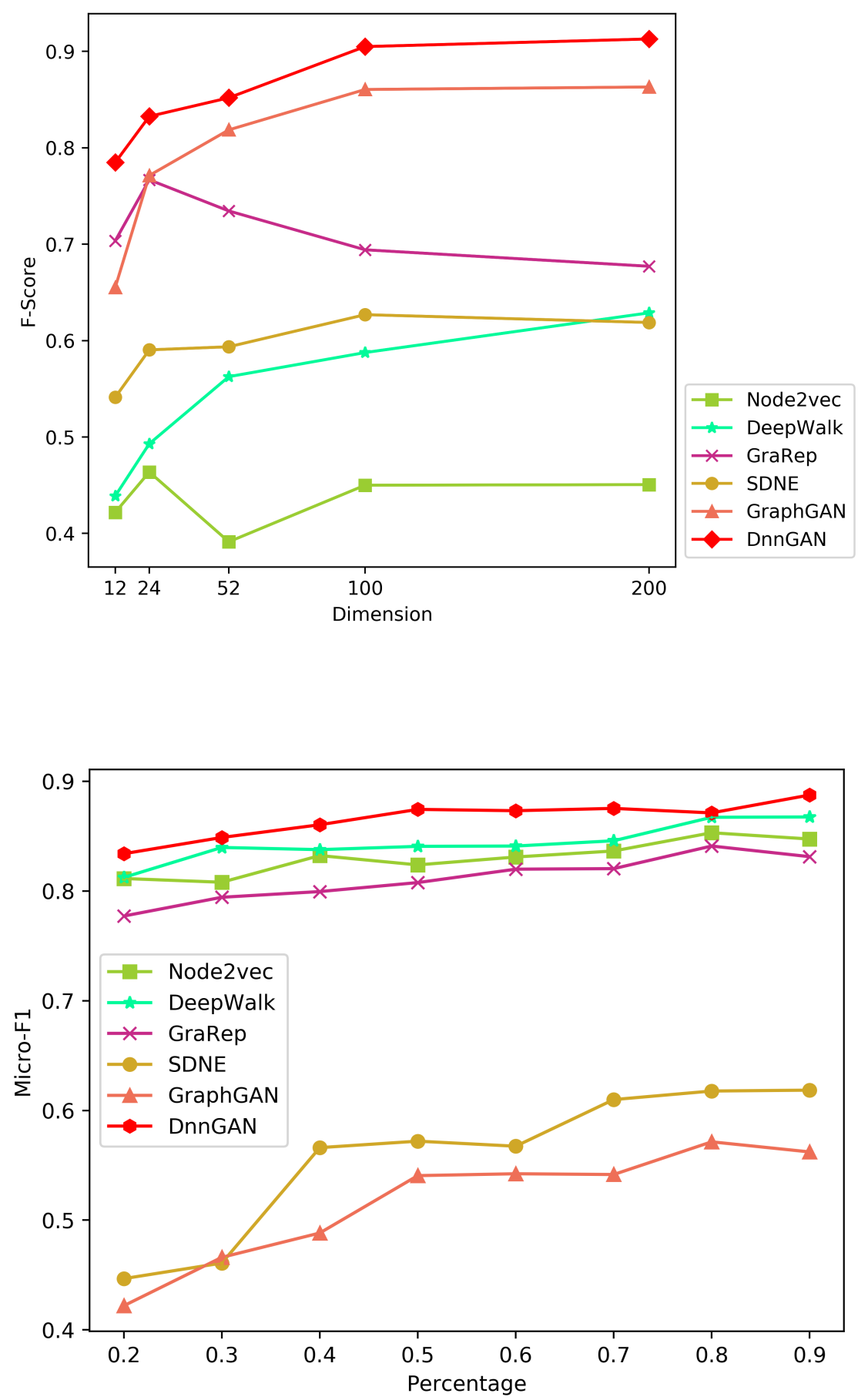

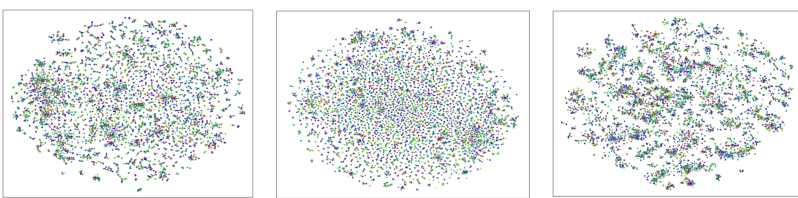

(a) Node2vec

(b) Line

c) Grarep

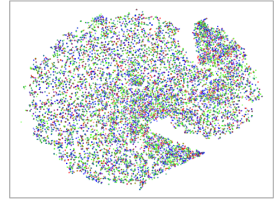

(d) SDNE

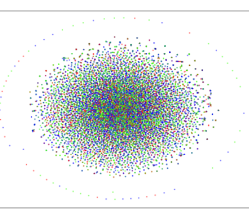

(e) MCNS

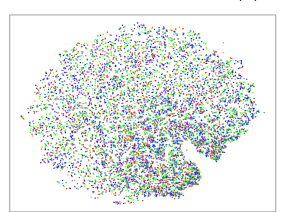

(f) GraphGAN

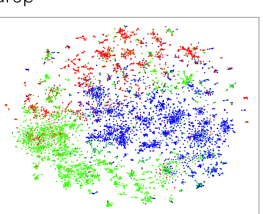

(g) DnnGAN 